\title{
Thin-disk laser pump schemes for large number of passes and moderate pump source quality
}

\author{
K. Schuhmann ${ }^{1,2, *}$, T. W. Hänsch ${ }^{3}$, K. Kirch ${ }^{1,2}$, A. Knecht ${ }^{2}$, F. Kottmann ${ }^{1}$, \\ F. Nez ${ }^{4}$, R. Pohl ${ }^{3}$, D. Taqqu ${ }^{1}$ and A. Antognini ${ }^{1,2}$ \\ ${ }^{1}$ Institute for Particle Physics, ETH, 8093 Zurich, Switzerland \\ ${ }^{2}$ Paul Scherrer Institute, 5232 Villigen-PSI, Switzerland \\ ${ }^{3}$ Max Planck Institute of Quantum Optics, 85748 Garching, Germany \\ ${ }^{4}$ Laboratoire Kastler Brossel, UPMC-Sorbonne Universites, CNRS, ENS-PSL \\ Research University, College de France, 4 place Jussieu, 75005 Paris, France
}

\author{
skarsten@phys.ethz.ch
}

\begin{abstract}
Novel thin-disk laser pump layouts are proposed yielding an increased number of passes for a given pump module size and pump source quality. These novel layouts result from a general scheme which bases on merging two simpler pump optics arrangements. Some peculiar examples can be realized by adapting standard commercially available pump optics simply by introducing an additional mirror-pair. More pump passes yield better efficiency, opening the way for usage of active materials with low absorption. In a standard multi-pass pump design, scaling of the number of beam passes brings about an increase of the overall size of the optical arrangement or an increase of the pump source quality requirements. Such increases are minimized in our scheme, making them eligible for industrial applications.
\end{abstract}

\section{Introduction}

The thin-disk laser [1, 2, 3] is a diode-pumped solid-state laser with high power and high pulse energy capabilities, high efficiency and excellent beam quality. The thin-disk laser active medium, depicted in Fig. 1 (a), is a thin disk with typical thickness of 100-500 $\mu \mathrm{m}$ and diameter up to a few $\mathrm{cm}$. Lasing and cooling occurs along the disk axis, while pumping is ensued in a quasi-end-pumped configuration. The rear side of the disk is acting as a high-reflective mirror (HR) for pump and laser wavelengths, and it is thermally coupled to a heat sink. Heat removal from the disk is efficient because of the large cooled surface to active volume ratio. Since the heat flux occurs along the laser axis, the thermally induced lens effects are minimized resulting in small phase-front distortions also for beams of large diameter $4,5,6,7$. This cooling scheme allows thus for power and energy scaling $8,9,10,11,12,13$ simply by increasing the diameter of laser and pump spots, eventually limited by amplified spontaneous emission effects [4, 14, 15, 16. Moreover, the efficient cooling allows pumping in the $\mathrm{kW}$ regime and the usage of quasi three-level-system materials having low quantum defect and high gain. To date, the paradigmatic material of choice, especially in industrial applications, is ytterbium-doped yttrium aluminium garnet (Yb:YAG) [17, 18, 19 but recently, researchers have concentrated on finding new materials with larger thermal conductivity for higher output power, and with larger bandwidth for ultrashort pulse generation or tunable lasers 9 , 20, 21, 22, 23, 24, 25. 
The small thickness of the disk guarantees excellent cooling and power scaling. Yet, in a single pass, only a small fraction of pump light is absorbed in the thin active medium. The light not absorbed in the disk in the first pass is reflected by the HR coating onto a second pass in the active medium. Even so, the absorption in the resulting double pass is too small. This shortcoming can be compensated using a multi-pass scheme for the pump light, that is, by redirecting the (not absorbed) pump beam into the disk several times [27].

A well defined pump region with sharp boundaries is fundamental, especially for three-level-system materials due to the high lasing threshold. To generate a pump profile which minimizes radial tails, on one hand the pump optics has to redirect the various passes at "exactly" the same position at the disk, and, on the other hand, each individual pump pass spot has to have sharp boundaries. Relay $4 \mathrm{f}$ telecentric imaging with unitary magnification is commonly used for this purpose [27] as its propagation matrix is exactly the negative unity matrix.

The scheme ordinarily used for disk laser pumping is first to homogenize a high-power diode laser by coupling it into either a multi-mode fiber or a rod homogenizer. The output face of this optical element is then imaged with suitable magnification onto the disk. The multi-pass propagation of the pump beam is realized using telecentric $4 \mathrm{f}$ relay imaging with unitary magnification, that is, by imaging the pump spot at the disk position from pass to pass without changing its size. The imaging properties of the $4 \mathrm{f}$ imaging scheme "per definition" guarantees that the beam spot profile and its divergence are exactly reproduced from pass to pass (neglecting phase-front distortions occurring at the disk).

Another practical advantage is given by the fact that $4 \mathrm{f}$ imaging can be realized with only a few optical elements also for a large number of passes. Commonly it is realized [27, 28, 29, 30, 31] using a parabolic mirror and a deflecting mirror system as shown in Fig. 1 (b) and (c). After collimation, the pump light from the homogenizer enters the pump optics and is being directed to the disk by the parabolic mirror. The light not absorbed in the disk in the first double-pass is reflected back to the parabolic mirror. At the parabolic mirror, the pump beam is collimated and sent to the mirror system (prisms) which after a double $90^{\circ}$ reflection redirects the light back onto the parabolic mirror, but at a different position. From there, the light proceeds to the disk for the second time. Iterating this scheme several times gives rise to a multi-pass pump pattern having several passes through the active medium.

The typical commercially available thin-disk pump modules [32, 33, 34] provide 24 or 48 passes 1 . A larger number of pump passes enables a reduction of the thin-disk thickness and doping concentration while keeping the same pump light absorption. The smaller heat resistance that results from reducing the thin-disk thickness brings about lower average temperature of the active medium which is advantageous for the three-level-systems. Moreover also thermal lens effects (spherical and aspherical components) of the thin-disk, are reduced leading to improved efficiency and beam quality. Similarly, a reduced doping ensues higher active medium thermal conductance due to decreased scattering of the active medium phonons on the doping ions yielding a more efficient cooling. The increase of pump passes also allows for the use of active medium materials with smaller absorption cross sections, and smaller absorption bandwidths.

\footnotetext{
${ }^{1}$ Throughout this paper, the number of passes $N$ is defined to be twice the number of reflections at the disk.
} 

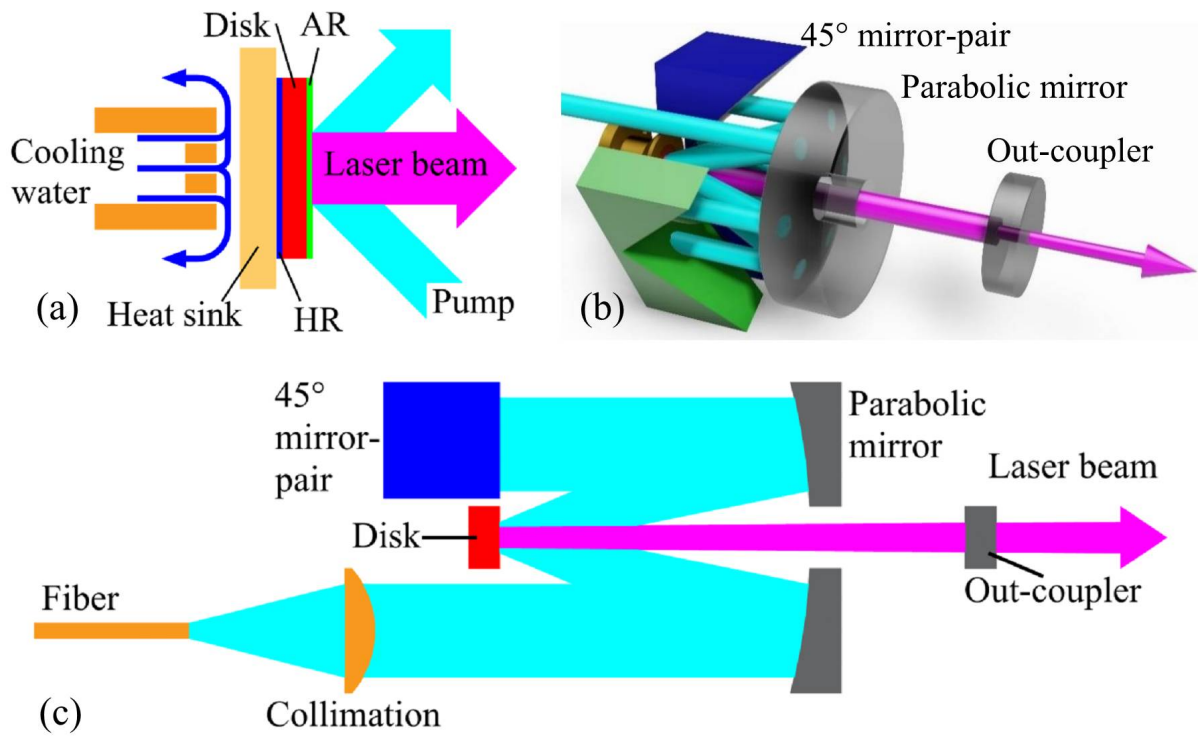

Figure 1: (Color online) Working principle of a thin-disk laser and thin-disk laser pump arrangement. (a) Scheme of the thin-disk active medium mounted on a water cooled heat sink. Lasing and cooling occurs along the disk axis. (b) $3 D$ schematic of the pump optics: heat sink (gold), parabolic mirror (gray), and prisms acting as mirror-pairs (green and blue). (c) Pump light multi-pass arrangement. The light from an homogenizer is imaged onto the disk via a parabolic mirror. The multi-pass is realized via the disk, the parabolic mirror and the $45^{\circ}$ mirror-pairs. In cyan is given the pump beam propagation in the multi-pass, in magenta the laser beam, HR and AR stand for high-reflector and anti-reflex layer, respectively.

However, such an increase of pass number has to be attained without increasing substantially the requirements for the pump beam quality expressed by the beam parameters product $P$ which is defined as the product of the pump beam's divergence angle (half-angle in the far-field) and beam waist (radius of the beam at its narrowest point).

In this paper, possible realizations of multi-pass pump layouts suited for thin-disk lasers are presented. We restrict the discussion to $4 \mathrm{f}$ imaging schemes. In Sec. 2 the $4 \mathrm{f}$ imaging and the multi-pass concepts are introduced with the help of an exemplary simple pump layout. Section 3 describes the state-ofthe-art of the standard commercially available thin-disk pump optics and its related pump beam propagation. The novel layouts we are proposing in this work, result from combining two multi-pass concepts: the first is based on the standard pump module design described in Sec. 3, the second is given in Sec. 4. The merging of these two concepts which is described in Sec. 5 enables the realization of multi-pass systems with large number of passes while only minimally increasing the complexity of the multi-pass system, the size of the optics, and the requirement for the pump source quality, making these schemes apt for industrial applications. A particular practical realization based on hexagonal mirror-pairs and hexagonal ordered fiber-coupled diodes is presented in Sec. 6 . 
followed by concluding remarks.

\section{A simple example of a multi-pass scheme based on 4 f relay imaging}

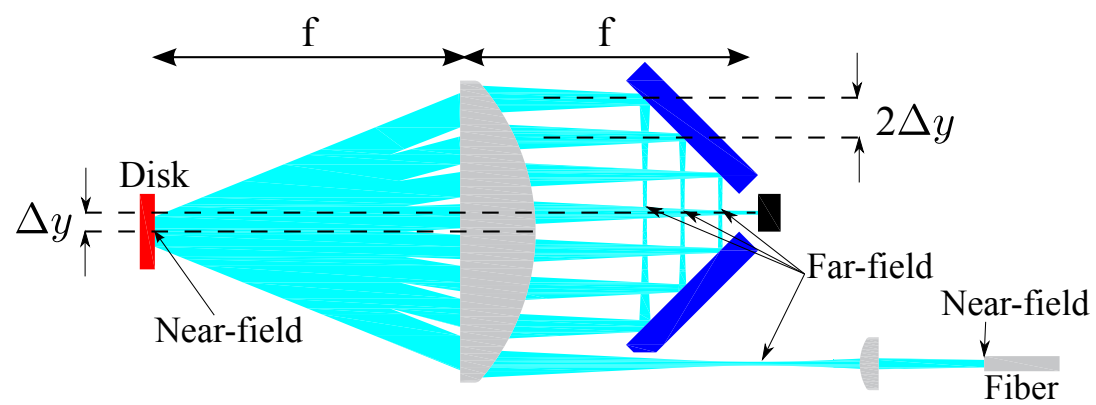

Figure 2: (Color online) Schematic of a $4 f$ multi-pass optical system realized by a $45^{\circ}$ mirror-pair (blue), a lens (gray) and a disk (red). The mirror-pair axis is shifted by $\Delta y$ relative to disk-lens axis. The pump beam multi-pass is given in cyan. The number of passes can be doubled by introducing a back-reflector (black).

A simple way to realize a multi-pass scheme based on $4 \mathrm{f}$ imaging is shown in Fig. 2 [35, 36]. The setup consists of a thin-disk, a lens with focal length $f$ and a pair of mirrors at $45^{\circ}$ angle relative to the optical axis. The spacings between disk and lens, and between lens and the intersect of the mirror-pair are $f$. Hence, the propagation from disk to mirror-pair can be seen as imaging from the disk image plane to the mirror-pair Fourier plane, and the full round-trip from disk to disk as a $4 \mathrm{f}$ relay imaging. In other words, the near-field laser profile at the disk is imaged by the lens into the far-field at the mirror-pair center and vice versa.

If the intercept (center) of the mirror-pair was located on the disk-lens axis, the light would propagate in this optical system in a closed loop as in a resonator. However, by displacing the mirror-pair center by $\Delta y$ as shown in the figure, a multi-pass configuration can be realized. The resulting far-field spacing of the various beam passes is $2 \Delta y$. The shift of the mirror-pair center relative to the common disk-lens axis breaks the symmetry which is necessary for the realization of a multi-pass scheme. The size of $\Delta y$ controls the number of achievable passes given constraints from the laser beam size, restrictions related with astigmatisms and the size of the various optical elements.

A common feature of multi-pass propagation is that the number of passes can be doubled by placing a back-reflector at its end causing the beam to retrace itself traveling in the opposite direction. For pump beams, the residual beam not absorbed in the disk after the back-and-forth propagation is consequently sent back to the homogenizer of the pump diodes. 


\section{State of the art of the commercial multi-pass pump systems}

A typical pump optics system for thin-disk lasers originally proposed in 27 and now available at TRUMPF, IFSW and Dausinger+Giesen is given in Fig. 11. It consists of a disk, a large parabolic mirror with focal length $f$ and two mirrorpairs (HR coated prism-pairs). Similar to the previous example, the distances between disk and parabolic mirror, and between parabolic mirror and prisms intersects are $f$. For the realization of a multi-pass propagation the symmetry of the optical layout has to be broken. Instead of shifting as previously the mirrorpair off-axis, in this case, the symmetry is broken by rotating one prism-pair by an angle $\phi_{1}$ relative to the other pair as depicted in Fig. 3.
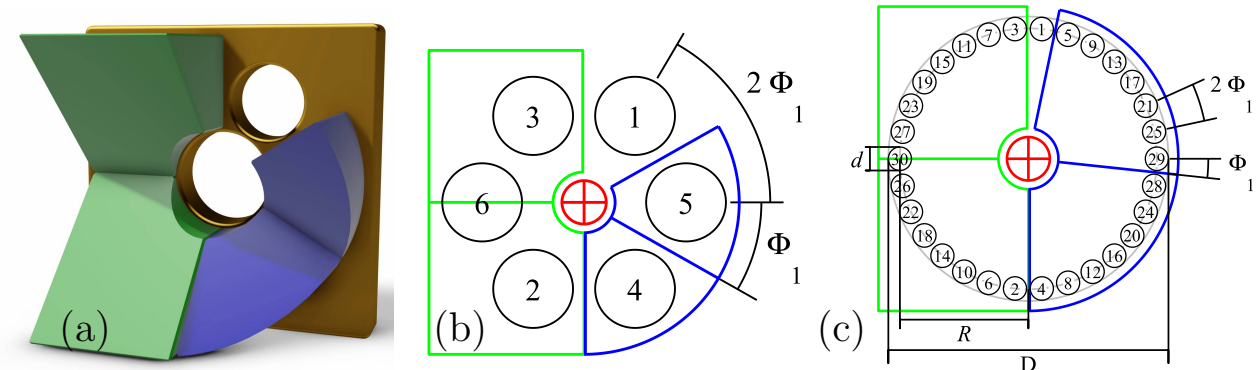

Figure 3: Schematics of the mirror-pairs and multi-pass beam routing at the parabolic mirror and mirror-pairs plane. (a) 3D arrangement of the mirror(prisms-) pairs commercially available. (b) Mirror-pairs contours and beam routing (which follows the given numbering) for 6 reflections (12 passes) at the disk. The disk position is given in red. The second mirror-pair (blue) is rotated by an angle $\phi_{1}=30^{\circ}$ relative to the first mirror-pair (green). At position 6 the beam is back-reflected doubling the number of passes. (c) Schematic for 30 reflections (60 passes) at the disk achieved by decreasing the angle $\phi_{1}$.

To understand the beam routing in the multi-pass pump optics consider Fig. 3 (b). The position of the pump beam at the mirror-pairs plane (and at the parabolic mirror as the collimated pump beams are parallel) is indicated by the numbering. The number 1 represents the position of the collimated in-coupled beam. After a reflection at the parabolic mirror the beam travels to the disk (red central circle). The remaining pump light reflected from the disk subsequently reaches the position 2 at the first mirror-pair after a second collimation at the parabolic mirror. From there the pump beam is redirected within the same mirror-pair to reach position 3 . Thereafter, it is sent towards the parabolic mirror, disk, parabolic mirror to reach the position 4 on the second mirror-pair. Via a path within this second mirror-pair the beam reaches position 5 where it is redirected again towards the disk (third reflection at the disk) and from there eventually to position 6 . At position 6 the beam is back-reflected giving rise to a propagation in opposite direction which results in a doubling of the number of pump passes on the disk.

The relative rotation of the mirror-pair intercepts by an angle $\phi_{1}$ gives rise to a rotational symmetric beam spot pattern with spot-to-spot angle of $2 \phi_{1}$. In this design, all far-field beams have the same radial distance from the optical 
axis resulting in a central region free of pump beam spots (near-field is used for beams at the disk, far-field for beams at the mirror-pairs plane, as defined in Fig. 2). Therefore, the mechanics holding the mirror-pair can have a central aperture as shown in Fig. 3 (a) in which the thin-disk can be placed. Similarly, the parabolic mirror can have a central aperture as shown in Fig. 1 (b) and (c) for laser beam access. In such a way, the radial symmetric beam pattern allows the usage of a parabolic mirror instead of a lens as in example of Fig. 2, which yields a folding of the beam propagation so that geometrically the mirror-pairs plane coincides with the disk plane. Thus, the utilization of a parabolic mirror reduces the size of the multi-pass pump optics, leads to smaller absorption losses and offers the possibility for elegant laser beam coupling (see off-axis aperture in Fig. $3(\mathrm{a})$ ).

The most natural way to increase the number of pump passes would be to reduce the angle $\phi_{1}$ as shown in Fig. 3 (c). The number of passes $N$ is given by $N=2 \times 180 / \phi_{1}$, where $\phi_{1}$ is expressed in degrees. The factor of 2 originates from the fact that each reflection corresponds to two passes in the active material. Furthermore, this equation assumes that the pump beam propagates back and forth in the multi-pass segment due to the back-reflector (placed e.g., at position 6 in Fig. 3 (b), or at position 30 in Fig. 3 (c)).

However, an increase of passes by decreasing the angle $\phi_{1}$, (assuming the same parabolic mirror size and focal length $f$ ) can be realized only by decreasing the far-field beam spot size. Overlapping of the far-field spots (spots at the mirror-pairs plane) is not acceptable because it implies aperture losses at the beam in-coupling. For large $N$ the maximal diameter $d$ of a single far-field beam spot, as can be seen in Fig. 3 , is given by $d=2 \pi R / N$, where $R$ is the radial distance of the pump beams from the optical axis at the parabolic mirror position. This implies $1 / N$ scaling of the pump beam parameters product and a poor usage of the mirror-pairs and parabolic mirror surfaces. On the contrary, if the total surface of the parabolic mirror was used, the diameter of the far-field spots would shrink approximately only as $1 / \sqrt{N}$.

A scheme which makes use of a much larger fraction of the parabolic mirror surface is presented in the Sec. 5. This scheme allows thus for scaling of the number of passes while only moderately increasing the demands on the quality of the pump source. Before describing this scheme, for didactic reasons in Sec. 4 a multi-pass layout is presented which forms one of the building blocks of the final schemes.

\section{Two mirror pairs whose intersects meet off- axis}

The generalized pump schemes we propose and that are detailed in next section are resting on two building blocks. The first one is given by the standard pump optics described in Sec. 3. The second one, described in this section, is a novel design realized also only with a disk, a parabolic mirror and two mirror-pairs as shown in Fig. 4.

Differently to the layout of Fig. 3, the second mirror-pair is rotated relative to the first one by an angle $\phi_{2}$ not around the disk axis but around an axis shifted by an offset $\Delta X$ as shown in the Fig. 4 (a). The resulting distribution of the far- 

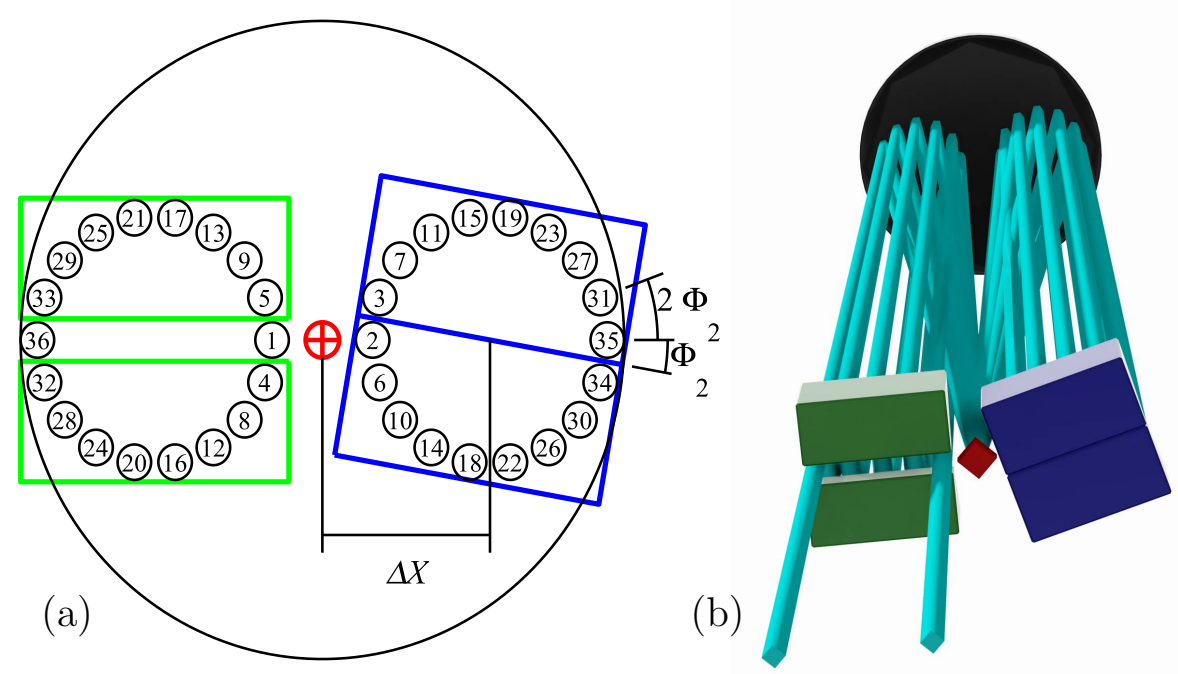

Figure 4: (a) Multi-pass configuration resulting from two mirror-pairs (prismpairs) whose intersects do not cross at the optical axis but at a point with $\Delta X$ offset. (b) Corresponding 3D drawings. Mirror-pairs are given in blue and green, the disk in red and the parabolic mirror in gray.

field spots is located on two separated circles. Similar to previous configurations, the number of passes is dictated by the angle of rotation, whereas the radius of these circles is given by the distance of the in-coupled beam to the center of the first mirror-pair depicted in green.

The beam is traveling between mirror-pairs, parabolic mirror and disk. The beam routing starts from the aperture (slit between the green mirror pair) at position 1 of the first mirror pair, and reaches position 2 at the second mirrorpair (blue) after a reflection at the disk and two reflections at the parabolic mirror. From position 2, the beam is redirected within the second mirrorpair to position 3. From position 3, it travels back to the first mirror-pair via a reflection at the disk and two reflections at the parabolic mirror, to reach position 4. Within the first mirror-pair the beam travels from position 4 to 5 , and from there it is sent again towards the disk. Iterating this scheme, several passes at the disk are realized, while the beam position at the mirror-pairs travels the given numbering.

For large number of passes, this multi-pass scheme requires large pump optics and shows a poor utilization of the parabolic mirror surface. Therefore, when used as shown in Fig. 4, it is not suited for the realization of a compact pump optics. However, when combined with other schemes as detailed in next section, layouts with more efficient usage of the parabolic surface can be realized. Still, this scheme as such (as in Fig. 4), can be used to design multi-pass laser amplifiers with large number of passes, because of the typically better beam parameter products of the laser beams and the less stringent size limitations. 


\section{Novel design with many more passes}

By merging the two concepts presented in Fig. 3 and Fig. 4, we can realize a novel multi-pass scheme with an increased number of passes while at the same time making efficient use of the surface of the parabolic mirror. Before proceeding to develop in a detailed way the ideas behind the novel configurations, the merging processes yielding the simple layout of Fig. 5 (d) is quickly introduced. The starting point is the setup of two mirror-pairs (green and blue) as in Fig. 5 (a) very similar to the setup in Fig. 3. Adding two more mirror-pairs (magenta and cyan) shown in Fig. 5 (b) results in an arrangement of four mirror-pairs as depicted in Fig. 5 (c) which can be simplified to the three-mirror-pair setup shown in Fig. 5 (d).

In more details, this merging proceeds in the following way. The panel (a) shows the same two mirror-pairs-arrangement as in Fig. 3 but contrarily to that situation, here the beam is not coupled in the center of region 1 but with a small displacement relative to the center of the region 1. Starting from this initial position, the pump beam travels in the pump optics following the given numbering at the positions indicated by the black dots, until it eventually reaches region 10. At this position, a third mirror-pair is placed, corresponding to the magenta mirror-pair of panel (b). This third mirror-pair reflects the beam within region 10, from the black dot to the black cross. From there, the beam undergoes a propagation indicated with the crosses back to region 1. At position 1 a fourth mirror-pair corresponding to the cyan mirror-pairs of the panel (b) is introduced, completing in this way the merging of the two concepts. The orientation of this forth mirror-pair is such to reflect the beam within region 1 at the position indicated with the empty square. Similar as before, starting from this position a propagation till the region 10 is followed. By iterating this scheme, a large number of passes on the disk can be realized.

Panel (c) of Fig. 5 displays the arrangement of all optical elements resulting from the merging of the two multi-pass schemes of panels (a) and (b). The pump beam propagation follows the indicated numbering yielding $N=2 \times n \times m=$ $2 \times\left(180 / \phi_{1}\right) \times\left(180 / \phi_{2}\right)$ passes, where $\phi_{1}$ and $\phi_{2}$ are the angular tilts in degrees of the second and forth mirror-pairs, respectively. Also in this case $N$ accounts for back-and-forth propagation in the multi-pass segment. The needed backreflection at position 50 in panel (c) is realized by the magenta mirror-pair. The numbers $n$ and $m$, which represent the number of reflections at the disk for the two separate multi-pass concepts, are used to classify the various configurations resulting from the merging process.

In this case, the third (magenta) mirror-pair was introduced just for didactic reasons: to better highlight the principle of the merging process. In fact, in this particular case its functionality can be realized by the first mirror-pair (see panel (d)), but this does not apply generally (see e.g. Fig. 6 (a)).

In conclusion, by adding only a small mirror-pair in the region of the incoupled beam (see Fig. 5 (d)), the number of pump passes relative to a standard pump optics design can be increased by a factor of $\left(180 / \phi_{2}\right)$. Moreover, the individual beam spots at the mirror-pair and at the parabolic mirror are larger than in the standard configuration assuming same parabolic mirror and number of passes, because of the better usage of the parabolic mirror surface. Hence, the demands for the pump source phase-space quality or pump optics size are decreased relative to the standard configurations. 

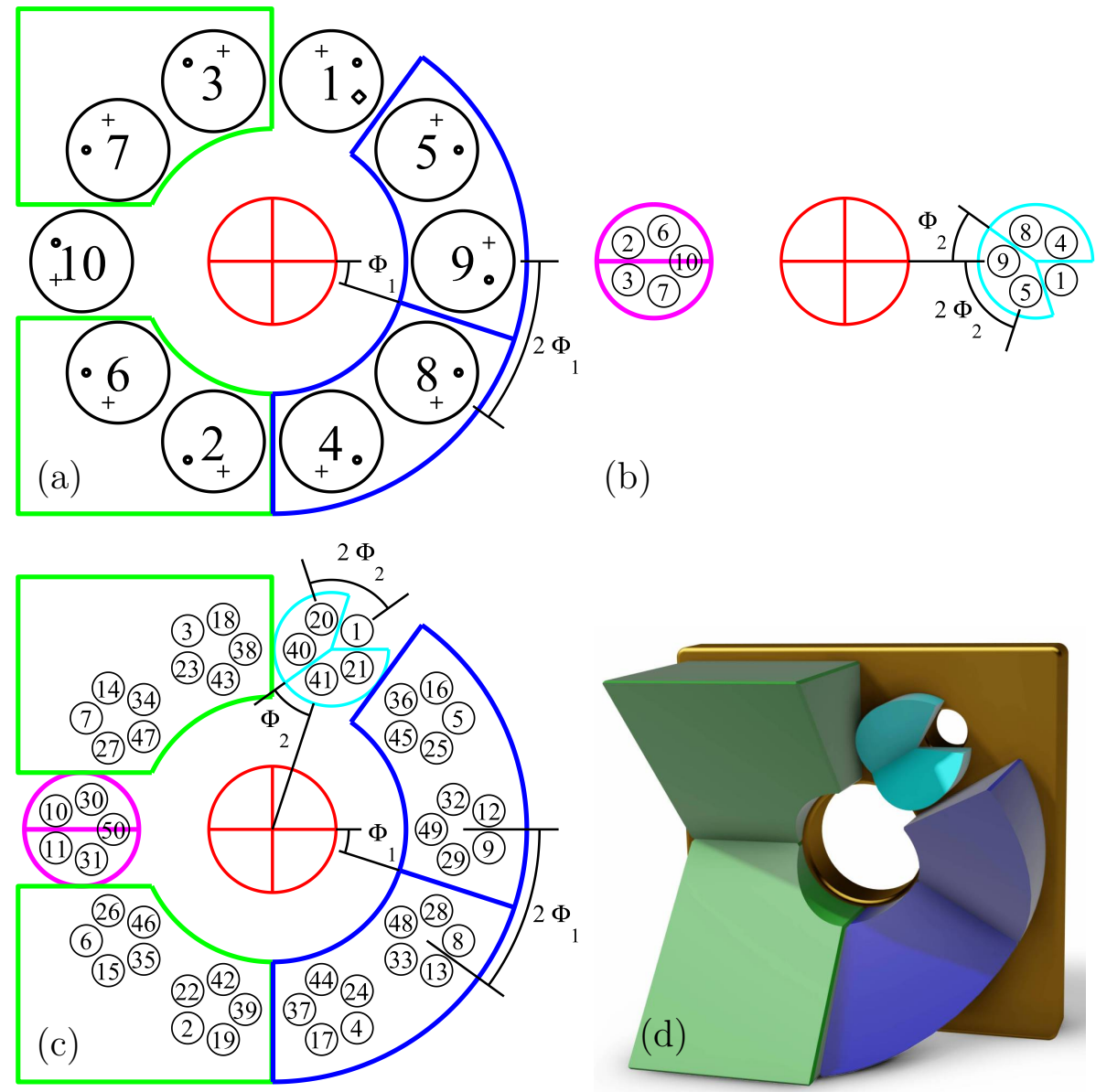

Figure 5: Principle of the merging of the two multi-pass schemes of Fig. 3 and Fig. 4. (a) Similar mirror-pair scheme as in Fig. 3 for 20 passes. The slightly different position of the in-coupled light in region 1 is producing the pattern indicated by the black dots at the various numbered regions. The crosses represent the beam position after the first reflection at the mirror-pair in region 10. (b) Similar mirror-pair scheme as in Fig. 4 for 20 passes. (c) The merging of the multi-passes scheme given in previous panels produces a beam propagation pattern of 100 passes. (d) $3 D$ representation of the 3 mirror-pairs needed to realize the 100 passes scheme of panel (c). The red circles represent the position of the disk, the blue, green, magenta and cyan contours represent the first, second, third and forth mirror-pairs, respectively. 
The merging described here can be interpreted as a generalization of the concept exploited already by TRUMPF [37] and shown in Fig. 6 (b). In our
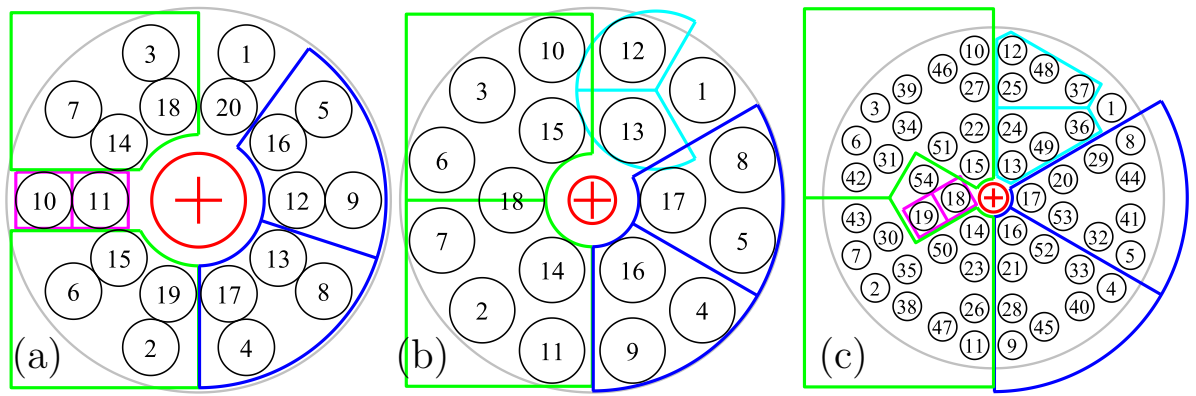

Figure 6: (a) $10 \times 2$ configuration yielding 40 passes. The third mirror pair in this case has been rotated by $90^{\circ}$ compared to the merging process described above. (b) $6 \times 3$ configuration yielding 36 passes as implemented by TRUMPF, and (c) $6 \times 3 \times 3$ configuration which results by iterating twice a merging process resulting in 108 passes.

scheme, the TRUMPF layout can be classified as a $n \times m=6 \times 3$ configuration leading to 36 passes in the disk. In panels (a) a $10 \times 2$ and in panel (c) a $6 \times 3 \times 3$ configuration is shown. The latter demonstrates that our merging processes can be iterated several times. Moreover our merging process can be applied for various initial configurations.

A comparison of the various configurations is shown in Fig. 7, where the "filling factor" $F$ is plotted against the number of pump passes. $F$ is defined as the ratio of the beam spots area at the parabolic mirror over the parabolic mirror area: $F=N d^{2} /\left(2 D^{2}\right)$, where $d$ is the individual beam spot diameter in the far-field and $D$ the parabolic mirror diameter (see Fig. 3 (c)). $F$ has been computed using the paraxial approximation. For the real situation, the "filling factor" differences between the various configurations are slightly larger due to the focal length increase of the parabolic mirror with increasing distance from the axis.

For the standard design of Fig. 3. $D=2 R+d$. For large $N, D \approx 2 R$ and $R \approx N d /(2 \pi)$ applies. Thus, in this limit the "filling factor" scales as $F \sim 1 / N$. For small $N$, these approximations do not apply and the "filling factor" increases with $N$ as well visible from the plot.

Similar behavior versus $N$ is found for other configurations. As a rule of thumb, with increasing $m$ the maximal "filling factor" is reached for larger values of $N$. The overall maximal "filling factor" is obtained for the $N=36$ design, resulting from the $6 \times 3$ configuration of TRUMPF depicted in Fig. 6 (b). For $N=108$, the configuration $6 \times 3 \times 3$ of Fig. 6] (c) shows by far the highest "filling factor".

Knowing the "filling factor" and the number of passes of a given configuration, it is possible to determine the minimal diameter $D$ of the parabolic mirror 


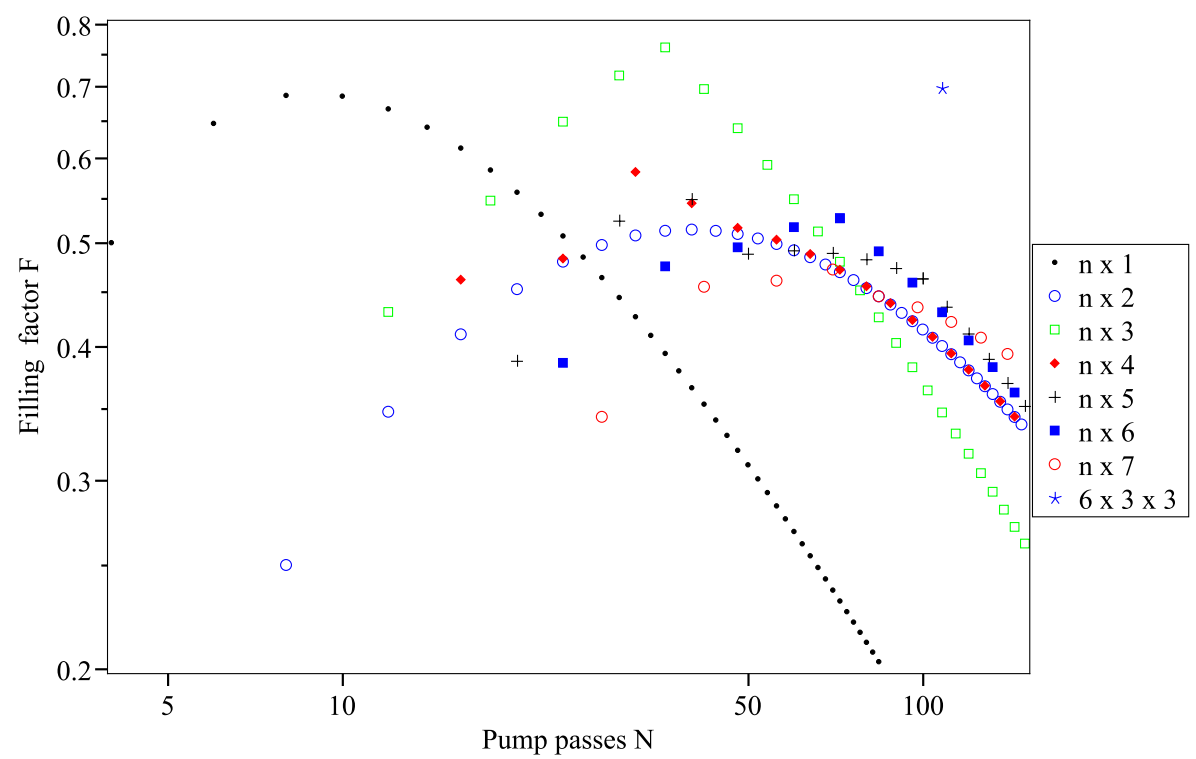

Figure 7: "Filling factor" $F$ for various configurations versus number of passes $N$ classified using the $n \times m$ nomenclature and computed using the paraxial approximation. The $n \times 1$ configuration represents the standard layout of Fig. 3 , the TRUMPF design corresponds to the maximum of the $n \times 3$ configuration and the star represents the $6 \times 3 \times 3$ configuration of Fig. 6 (c).

and the maximal beam parameters product $P$ of the pump source:

$$
\begin{aligned}
D & >\sqrt{\frac{8 N}{F}} \frac{P f}{d_{\text {pump }}} \\
P & <\sqrt{\frac{F}{8 N}} \frac{D d_{\text {pump }}}{f}
\end{aligned}
$$

where $d_{\text {pump }}$ is the pump spot diameter at the disk and $f$ the focal length of the parabolic mirror.

The optimal scheme that is used in each particular application is a trade-off between the number of passes needed, pump module size and the quality and costs of the available pump source.

Another aspect that must be considered is the misalignment sensitivity of the pump optics and the related required manufacturing precision. Figure 8 shows misalignment plots for various pump schemes. For an ideal alignment, each pump spot perfectly overlaps in the center of the disk. However practically, deviations from the ideal situation occur: for example the mirrors forming the mirror-pairs may not be exactly orthogonal to each other, or the mirror-pair intersects may not be perfectly orthogonal to the symmetry axis, etc. These misalignments give rise to deviations of the various pump spots from the center of the disk. Due to the rotation of the pump beam, compensation occurs while propagating in the standard pump optics, so that the deviations of the pump spots from the ideal position first increase and then decrease as indicated by the numbering in Fig. 8. As the average deviation grows with the number of passes 


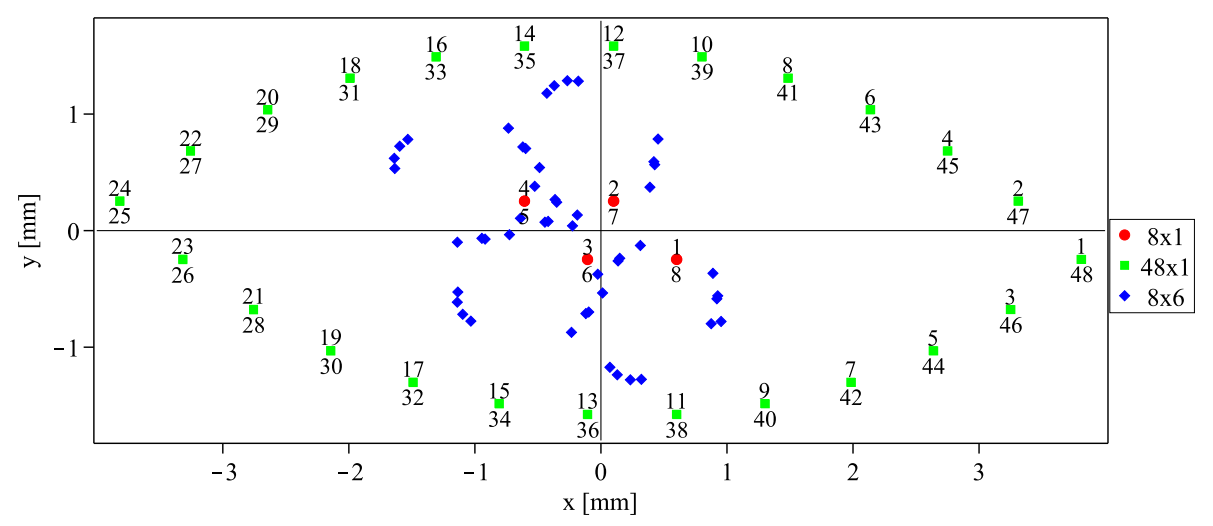

Figure 8: (Color online) Example of misalignment plot showing the deviation of the various pump beam passes at the thin-disk position for a departure of the angle between the two mirrors forming the first mirror-pair (given in green in all figures) by 2.5 mrad from 90 degrees. The parabolic mirror has a focal length of $200 \mathrm{~mm}$. The red points represent the deviation for the various passes in the standard $8 \times 1$ design with $N=16$, the green squares for the standard $48 \times 1$ design with $N=96$, and the blue diamonds for our $8 \times 6$ configuration also with $N=96$. The numbering which for clarity has been applied only to the standard configurations represents the pass number along the pump beam propagation. Similar plots are obtained for other misalignements.

$N$, well visible by comparing the red circles with the green squares, misalignment effects becomes more severe for pump optics with large number of passes. However, in our design the large number of passes is reached starting from a standard design with small number of passes. Thus the deviations caused by misalignments are mitigated in our schemes compared with the standard design having the same number of passes, so that the presently used manufacturing precision is sufficient.

\section{A particular example based on a $6 \times 6$ config- uration with triangular input aperture}

A realization of a 72 passes pump optics resulting from a $6 \times 6$ configuration is presented in Fig. 9. Two large hexagonal mirror-pairs, two small mirror-pairs and a flat end-mirror are used in this configuration. The hexagonal mirrors, placed as shown in Fig. 9 (a) and (b), naturally give rise to the central aperture necessary for the laser beam without the need of intensive manufacturing related with "internal" cutaways as e.g., necessary for the prisms of Fig. 5 (d). The beam losses occurring at position 6 of Fig. 3 (b) or at position 50 of Fig. 5 (c) at the mirror-pair intersects are not present in this configuration because at position 36 we are using simply a flat mirror as back-reflector 2 qualifying this scheme for improved efficiency and power scaling.

${ }^{2}$ When high power is requested, frequently the commercially available pump optics based on Fig. 3 (b) design, at position 6 (or equivalent positions) are equipped with a back-reflector to avoid losses which would be caused by the mirror-pair intersect. 

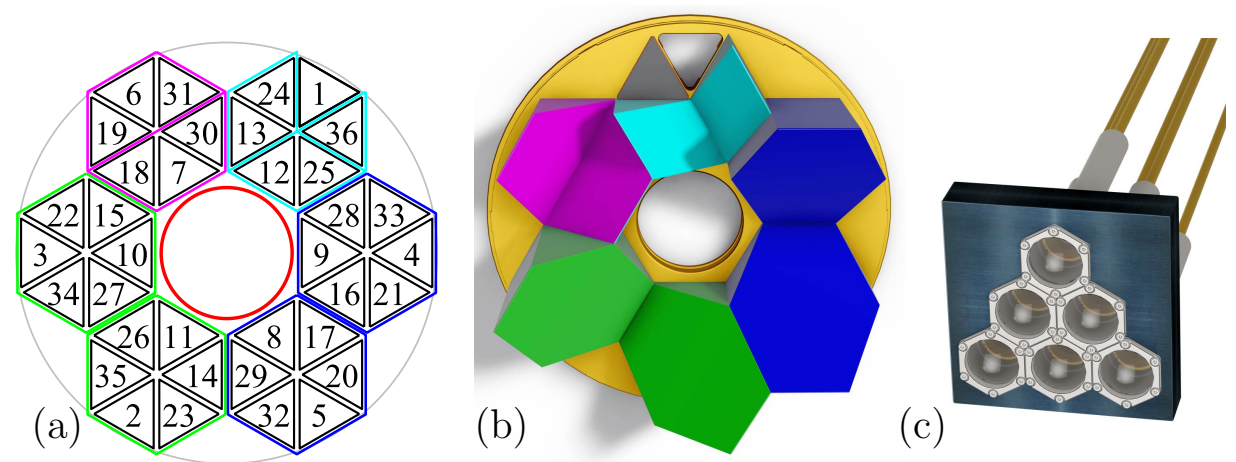

Figure 9: (a) $6 \times 6$ configuration yielding 72 passes based on hexagonal mirrorpairs. (b) $3 D$ drawings of the mirror-pairs. This hexagonal shaped mirror-pairs are particularly suited for a triangular illumination which can be formed by merging several collimated beams arranged on an hexagonal lattice as shown in (c) and imaging them after appropriate magnification at the input beam triangular aperture (at position 1).

The triangular input aperture, which is successively imaged on the various hexagonal mirrors seems strange for pump optics design since the profile of commonly used homogenized pump radiation is usually rotationally symmetric. However, when high pump power is required, several collimated parallel beams could be combined to illuminate the triangular input aperture. The most homogeneous way to illuminate the triangular input aperture is achieved by combining $3,6,10,15 \ldots$ pump diode homogenizers outputs, placed on an hexagonal grid as shown in Fig. 9 (c). Even though at the input-beam aperture (far-field) the various parallel beams are located at different positions, the parabolic mirror merges them into a single round spot at the disk position. Large pump power density at the disk can be reached in this way, with pump diameter given by the individual pump source outputs characteristics (beam parameters product $P)$.

\section{Conclusions}

A general scheme for multi-pass $4 \mathrm{f}$ pump optics suited for thin-disk lasers has been presented here, which can accommodate for large number of passes while keeping the requirements for the pump source beam quality moderate. A particular realization of this general scheme can be simply achieved by inserting a small additional mirror pair at the input beam position of a standard pump design. In such a way, the same pump optics can be operated, on one hand, without this new additional mirror-pair, for example, for high energy pulsed pumping and thick disks. On the other hand, by inserting a mirror-pair at the in-coupling position tilted by 90,60 and 30 degrees, the number of passes can be increased by factors of 2,3 and 6 , respectively, for the case that larger numbers of passes are needed. Thus, it is straightforward to adapt the number of passes to the given pump source quality and active medium properties. We also presented some simple formulae which can be used to estimate the maximal beam parameters product a pump source must have, or the minimal parabolic mirror 
diameter given a certain configuration and number of passes.

The increased number of passes achievable by using the schemes presented here, which is particularly important for active media having small single pass absorption (due to lower disk thickness, lower doping, lower active medium absorption cross section, and smaller absorption bandwidth), yields an increased efficiency, enabling power scaling, lower lasing threshold and the usage of novel materials.

This work is supported by the SNF_200021L-138175, the SNF_200020_159755 and the ERC StG. \#279765.

\section{References}

[1] A. Giesen, H. Hügel, A. Voss, K. Wittig, U. Brauch, H. Opower, "Scalable concept for diode-pumped high-power solid-state lasers", Applied Physics B 58, 365-372 (1994).

[2] U. Brauch, A. Giesen M. Karszewski, Chr. Stewen and A. Voss, "Multiwatt diode-pumped Yb:YAG thin disk laser continuously tunable between 1018 and $1053 \mathrm{~nm}$ ", Opt. Lett. 20, 713-715 (1995).

[3] C. Stewen, K. Contag, M. Larionov, A. Giesen, and H. Hügel, "A 1-kW CW thin disc laser", IEEE J. Sel. Top. Quantum Electron. 6, 650-657 (2000).

[4] A. Antognini, K. Schuhmann, F.D Amaro et al., "Thin-Disk Yb:YAG Oscillator-Amplifier Laser, ASE, and Effective Yb:YAG Lifetime," Journal of Quantum Electronics, IEEE 45, 993-1005 (2009).

[5] J. Speiser and A. Giesen, "Numerical Modeling of High Power ContinuousWave Yb:YAG Thin Disk Lasers, Scaling to 14 kW", Advanced Solid-State Photonics, WB9 (2007).

[6] G. Zhu, X. Zhu, M. Wang, Y. Feng and C. Zhu, "Analytical model of thermal effect and optical path difference in end-pumped Yb:YAG thin disk laser", Appl. Opt. 53, 6756-6764 (2014).

[7] J. Perchermeier and U. Wittrock, "Precise measurements of the thermooptical aberrations of an Yb:YAG thin-disk laser", Opt. Lett. 38, 24222424 (2013).

[8] J. Speiser, "Thin disk laser-energy scaling", Laser Physics 19, 274-280 (2009).

[9] A. Giesen and J. Speiser, "Fifteen Years of Work on Thin-Disk Lasers: Results and Scaling Laws", Selected Topics in Quantum Electronics 13, 598-609 (2007).

[10] S. Piehler, B. Weichelt, A. Voss, M. Abdou Ahmed and Thomas Graf, "Power scaling of fundamental-mode thin-disk lasers using intracavity deformable mirrors", Opt. Lett. 37, 5033-5035 (2012).

[11] J. Mende, E. Schmid, J. Speiser, G. Spindler and A. Giesen, "Thin disk laser: power scaling to the $\mathrm{kW}$ regime in fundamental mode operation", Proc. SPIE 7193, Solid State Lasers XVIII: Technology and Devices, 71931V (2009). 
[12] H. Fattahi, H.G. Barros, M. Gorjan et al., "Third-generation femtosecond technology", Optica 1, 45-63 (2014).

[13] C.J. Saraceno, F. Emaury, C. Schriber, A. Diebold, M. Hoffmann, M. Golling, T. Südmeyer and U. Keller, "Toward Millijoule-Level High-Power Ultrafast Thin-Disk Oscillators", Selected Topics in Quantum Electronics 21, 106-123 (2015).

[14] J. Speiser, "Scaling of thin-disk lasers-influence of amplified spontaneous emission", J. Opt. Soc. Am. B 26, 26-35 (2009).

[15] H. Furuse, H. Chosrowjan, J. Kawanaka, N. Miyanaga, M. Fujita and Y. Izawa, "ASE and parasitic lasing in thin disk laser with anti-ASE cap", Optics Express 21, 13118 (2013).

[16] P. Peterson, A. Gavrielides, T.C. Newell, N. Vretenar and W.P. Latham, "ASE in thin disk lasers: theory and experiment", Optics Express 19, 25672 (2011).

[17] TRUMPF GmbH, www.trumpf-laser.com/de/produkte/festkoerperlaser/scheibenlaser/trudisk.html

[18] T. Gottwald, C. Stolzenburg, D. Bauer, J. Kleinbauer, V. Kuhn, T. Metzger, S. Schad, D. Sutter, and A. Killi, "Recent disk laser development at TRUMPF", Proc. SPIE 8547, 85470C (2012).

[19] M. Larionov and J. Neuhaus "Regenerative thin disk amplifier with a pulse energy of $120 \mathrm{~mJ}$ at $1 \mathrm{kHz}$ ", Advanced Solid State Lasers, ATh2A.51 (2014).

[20] R. Peters, C. Kränkel, K. Petermann and G. Huber, "Broadly tunable highpower $\mathrm{Yb}: \mathrm{Lu}_{2} \mathrm{O}_{3}$ thin disk laser with $80 \%$ slope efficiency", Opt. Express 15(11) 7075-7082 (2007).

[21] R. Peters, "Ytterbium-doped sesquioxides as highly efficient laser materials", ISBN: 978-3-8322-8504-3, Shaker Verlag (2009).

[22] M. Siebold, M. Loeser, F. Roeser, M. Seltmann, G. Harzendorf, I. Tsybin, S. Linke, S. Banerjee, P. D. Mason, P. J. Phillips, K. Ertel, J. C. Collier, and U. Schramm, "High-energy, ceramic-disk Yb:LuAG laser amplifier", Opt. Express 20, 21992-22000 (2012).

[23] C.J. Saraceno, C. Schriber, F. Emaury, O.H. Heckl, C.R.R Baer, M. Hoffmann, K. Beil, C. Kränkel, M. Golling, T. Südmeyer and U. Keller, "Cutting-Edge High-Power Ultrafast Thin Disk Oscillators", Applied Sciences 3, 355-395 (2013).

[24] S. Ricaud, A. Jaffres, P. Loiseau, B. Viana, B. Weichelt, M. Abdou-Ahmed, A. Voss, T. Graf, D. Rytz, M. Delaigue, E. Mottay, P. Georges and F. Druon, "Yb:CaGdAlO 4 thin-disk laser", Opt. Lett. 36, 4134-4136 (2011).

[25] B. Dannecker, X. Délen, K.S. Wentsch, B. Weichelt, C. Hönninger, A. Voss, M. Abdou Ahmed and T. Graf, "Passively mode-locked $\mathrm{Yb}: \mathrm{CaF}_{2}$ thin-disk laser", Opt. Express 22, 22278-22284 (2014). 
[26] A. Giesen, J. Speiser, R. Peters, C. Kränkel, and K. Petermann, "Thin-disk lasers come of age", Photonics Spectra 41, 52-58 (2007).

[27] S. Erhard, M. Karszewski, C. Stewen, A. Giesen, K. Contag, and A. Voss, "Pumping schemes for multi-kW thin disk lasers", in ASSL(OSA, Davos, Switzerland), OSA TOPS 34, 7884 (2000).

[28] K. Contag, S. Erhard, A. Giesen, M. Karszewski, C. Stewen, A. Voss, "Laser amplifying system", DE Patent App. DE1998135108 (2000).

[29] S. Erhard, A. Giesen, M. Karszewski, C. Stewen and A. Voss, "Laser amplifying system", DE Patent App. DE1998135107 (2000).

[30] S. Erhard, A. Giesen and C. Stewen, "Laser amplifying system", DE Patent App. DE2000105195 (2001).

[31] S. Erhard and A. Giesen, "Laser amplifying system", DE Patent App. DE2000105194 (2001).

[32] Dausinger + Giesen GmbH, Stuttgart, http://www.dausingergiesen.de/sites/default/files/TDM 1.0 Lab_en_0.pdf.

[33] Dausinger + Giesen GmbH, Stuttgart, http://www.dausingergiesen.de/sites/default/files/TDM 2.0 HP Lab.pdf.

[34] Institut für Strahlwerkzeuge, Stuttgart, http://www.ifsw.unistuttgart.de/produkte/pdf/1406_IFSW_HO_TDPumpModules_end.pdf.

[35] A. Giesen, M. Larionov and K. Schuhmann, "Laserverstärkersystem", DE Patent App. DE201110075274 (2012).

[36] Dausinger + Giesen GmbH, Stuttgart, http://www.dausingergiesen.de/sites/default/files/TDM 005_4.pdf.

[37] A. Killi and S. Schad "Pump light assembly for a disk laser", DE Patent App. DE201110004204 (2012). 